\title{
Shifted ultraspherical pseudo-Galerkin method for approximating the solutions of some types of ordinary fractional problems
}

\author{
Mohamed Abdelhakem ${ }^{1,2}$ (D), Doha Mahmoud ${ }^{1}$, Dumitru Baleanu ${ }^{3,4,5^{*}}$ (D) and Mamdouh El-kady ${ }^{1,2}$ (D)
}

\author{
"Correspondence: \\ dumitru@cankaya.edu.tr \\ ${ }^{3}$ Department of Mathematics, \\ Cankaya University, Ankara, Turkey \\ ${ }^{4}$ Institute of Space Sciences, \\ Magurele-Bucharest, Romania \\ Full list of author information is \\ available at the end of the article
}

\begin{abstract}
In this work, a technique for finding approximate solutions for ordinary fraction differential equations (OFDEs) of any order has been proposed. The method is a hybrid between Galerkin and collocation methods. Also, this method can be extended to approximate fractional integro-differential equations (FIDEs) and fractional optimal control problems (FOCPs). The spatial approximations with their derivatives are based on shifted ultraspherical polynomials (SUPS). Modified Galerkin spectral method has been used to create direct approximate solutions of linear/nonlinear ordinary fractional differential equations, a system of ordinary fraction differential equations, fractional integro-differential equations, or fractional optimal control problems. The aim is to transform those problems into a system of algebraic equations. That system will be efficiently solved by any solver. Three spaces of collocation nodes have been used through that transformation. Finally, numerical examples show the accuracy and efficiency of the investigated method.
\end{abstract}

Keywords: Shifted ultraspherical polynomials; Fractional differential equations; Fractional integro-differential equations; Fractional optimal control problems; Galerkin method; Spectral method; Error analysis

\section{Introduction}

OFDEs play a role in many branches and applications. These applications can be found in risk theory [1], physics [2], biological phenomena, and diseases [3-6]. Also, the applications of FIDEs have been presented for electromagnetic [7], microelectronics [8], and hematopoietic stem cell modeling [9]. Moreover, many models have been constructed using FOCPs $[10,11]$. For that reason, the importance of fractional calculus has emerged.

Most of these problems and models have no exact solutions. Consequently, the researchers focus on investigating and developing new numerical methods. For OFDEs, in [12] the authors presented a spectral method using shifted Chebyshev polynomials (SCHPs) of the second kind. While in [13], the same authors used the same technique but with the sixth kind of SCHPs. A different technique by using a nonpolynomial spline function was employed in [14, 15]. The second kind Wright function with Erdélyi-Kober fractional derivatives was used in [16]. Similar techniques were used for approximating

(c) The Author(s) 2021. This article is licensed under a Creative Commons Attribution 4.0 International License, which permits use, sharing, adaptation, distribution and reproduction in any medium or format, as long as you give appropriate credit to the original author(s) and the source, provide a link to the Creative Commons licence, and indicate if changes were made. The images or other third party material in this article are included in the article's Creative Commons licence, unless indicated otherwise in a credit line to the material. If material is not included in the article's Creative Commons licence and your intended use is not permitted by statutory regulation or exceeds the permitted use, you will need to obtain permission directly from the copyright holder. To view a copy of this licence, visit http://creativecommons.org/licenses/by/4.0/ 
FIDEs. Alternative Legendre functions in [17] were used to set up an operational matrix by the collocation method. In [9], the authors used smoothed pseudo-splines refinable functions to construct Riesz wavelets. And as a direct development of the mentioned methods and algorithms, several methods have been formulated to approximate FOCPs. For details about those methods, refer to $[10,18,19]$.

The spirit in using the spectral method is the choice of the basis function [20,21]. The smoothness properties of the basis functions control the decay rate of the coefficients of expansion. The boundary conditions have no effect on the rate. Unlike the other orthogonal polynomials, ultraspherical polynomials (UPs) are not nearly as popular in use as basis functions [22-24]. However, UPs motivate our interest because they include Chebyshev and Legendre polynomials and some other polynomials as subclasses of them [25].

The importance of the Galerkin method (GM) is that, for the presented technique, it can be used for the solution of a wide class of ordinary fractional problems (OFPs). Throughout this work, OFPs are OFDEs, FIDEs, or OCFPs. One of the advantages of GM is that it is not a difficult method. On the other hand, every equation has its own algorithm. The presented technique is a mix of two well-known spectral methods: collocation method [26-28] and Galerkin method [29-31]. We call that method pseudo-Galerkin method (pseudo-GM).

The work is coordinated as follows: in Sect. 2, we introduce Caputo's fractional derivative. Then, UPs (SUPs) and some of their properties are presented. Finally, the notion of integration matrix (B-matrix) is presented. In Sect. 3, the main results of the paper are stated and proved. Those results give formulae that assert the derivatives of SUPs and the spectral expansion. In Sect. 4, the approximate of OFPs is stated by employing shifted ultraspherical pseudo-GM (SU-pseudo-GM). Then, the error analysis and upper bound for the expansion are estimated in Sect. 5. The correctness and effectiveness of SU-pseudoGM are proved by different types of OFPs in Sect. 6. The last section "7" contains the conclusion and remarks.

\section{Preliminaries and notations}

This section presents some core definitions and concepts needed throughout this paper. We shall begin with the well-known Caputo fractional derivative for the function $\phi(x)$ of order $\delta$ denoted by $D^{\delta}(\phi(x))$ :

$$
D^{\delta}(\phi(x))=\frac{1}{\Gamma(r-\delta)} \int_{0}^{x}(x-s)^{r-\delta-1} \frac{d^{r} \phi(s)}{d s^{r}} d s,
$$

where $r-1 \leq \delta<r, r \in \mathbb{N}$.

As a direct result of Eq. (1), we have

$$
D^{\delta} x^{t}= \begin{cases}0, & t \in \mathbb{N} \cup\{0\} \text { and } t<\lceil\delta\rceil, \\ \frac{\Gamma(t+1)}{\Gamma(t+1-\delta)} x^{t-\delta}, & t \in \mathbb{N} \cup\{0\} \text { and } t \geq\lceil\delta\rceil \text { or } t \notin \mathbb{N} \text { and } t>\lfloor\delta\rfloor .\end{cases}
$$

Besides, one of the advantages of Caputo's fractional derivative is the linearity property. For any two arbitrary constants $C_{1}$ and $C_{2}$,

$$
D^{\delta}\left(C_{1} \phi_{1}(x)+C_{2} \phi_{2}(x)\right)=C_{1} D^{\delta}\left(\phi_{1}(x)\right)+C_{2} D^{\delta}\left(\phi_{2}(x)\right) .
$$

For more details about fraction derivatives, refer to the review [32]. 
Proceeding with the fundamental concepts, some properties of UPs $\left(\mathcal{U}_{j}^{\nu}(x)\right)$ and SUPs $\left(\mathcal{U}_{j}^{* v}(x)\right)$, of degree $j$ and real parameter $v>-\frac{1}{2}$, will be presented. UPs are the special case of Jacobi polynomials with analytic form [25]:

$$
\mathcal{U}_{j}^{v}(x)=\sum_{r=0}^{\lfloor j / 2\rfloor}(-1)^{r} \frac{\Gamma(j-r+v)}{\Gamma(v) \Gamma(r+1) \Gamma(j-2 r+1)}(2 x)^{j-2 r}, \quad x \in[-1,1], j=0,1,2, \ldots
$$

As special cases: $L_{j}(x)=\mathcal{U}_{j}^{0.5}(x)$ and $\mathcal{T}_{j}(x)=\frac{j}{2} \lim _{\nu \rightarrow 0} \frac{\mathcal{U}_{j}^{\nu}(x)}{v}, j \geq 1$, where $L_{j}(x)$ and $\mathcal{T}_{j}(x)$ are Legendre and Chebyshev polynomials, respectively.

Also, UPs can be generated from Rodrigueś formula [33]

$$
\mathcal{U}_{j}^{v}(x)=\frac{1}{(-2)^{j}(j !)} \frac{\Gamma\left(v+\frac{1}{2}\right) \Gamma(j+2 v)}{\Gamma(2 v) \Gamma\left(j+v+\frac{1}{2}\right)}\left(1-x^{2}\right)^{\frac{1}{2}-v} \frac{d^{j}}{d x^{j}}\left(1-x^{2}\right)^{j+v-\frac{1}{2}}
$$

while SUPs, defined on the interval [0,1], can be obtained from [34]

$$
\mathcal{U}_{j}^{* v}(x)=\frac{2(v+j-1)(2 x-1) \mathcal{U}_{j-1}^{* v)}(x)-(2 v+j-2) \mathcal{U}_{j-2}^{* v}(x)}{j}, \quad j=2,3, \ldots,
$$

where $\mathcal{U}_{0}^{* v}(x)=1$ and $\mathcal{U}_{1}^{* v}(x)=2 v(2 x-1)$.

Or in terms of its derivatives [35]

$$
\mathcal{U}_{j}^{* v}(x)=\frac{1}{2(j+v)}\left[\frac{2 v+j}{j+1} D \mathcal{U}_{j+1}^{* v}(x)-\frac{j}{2 v+j-1} D \mathcal{U}_{j-1}^{* v}(x)\right], \quad j=2,3, \ldots
$$

SUPs at the boundaries are as follows:

$$
\mathcal{U}_{j}^{* v}(0)=(-1)^{j} \frac{\Gamma(j+2 v)}{\Gamma(j+1) \Gamma(2 v)}, \quad \mathcal{U}_{j}^{* v}(1)=\frac{\Gamma(j+2 v)}{\Gamma(j+1) \Gamma(2 v)}, \quad j=0,1,2, \ldots
$$

The set $\left\{\mathcal{U}_{j}^{* v}(x)\right\}_{j}$ forms an orthogonal set w.r.t. the weight function $w^{\nu}(x)=\left(x-x^{2}\right)^{\nu-\frac{1}{2}}$ :

$$
\int_{0}^{1} w^{v}(x) \mathcal{U}_{r}^{* v}(x) \mathcal{U}_{s}^{* v}(x) d x= \begin{cases}0, & r \neq s \\ \psi_{r}^{v}, & r=s\end{cases}
$$

where $\psi_{r}=\frac{\pi 2^{1-4 v} \Gamma(r+2 v)}{(r+v) \Gamma(r+1)(\Gamma(v))^{2}}$.

Finally, the expression B-matrix will be presented. This matrix will be used for solving FIDEs and FOCPs. According to the spectral method, let $\phi(x)$ be a differential function over the interval $[0,1]$. Then $\phi(x)$ can be approximated using $M+1$ points as follows [36]:

$$
\phi(x)=\sum_{m=0}^{M} c_{m} \mathcal{B}_{m} \mathcal{T}_{m}^{*}(x)
$$

where

$$
\mathcal{B}_{m}=\frac{2}{M} \sum_{r=0}^{M} c_{r} \phi\left(x_{r}\right) \mathcal{T}_{m}^{*}\left(x_{r}\right)
$$


such that: $\mathcal{T}_{m}^{*}(x)$ is the SCHP of degree $m, c_{0}=c_{M}=0.5$ and $c_{m}=1$ for $m=1,2, \ldots, M-1$, $x_{r}=0.5 *\left(1-\cos \frac{r \pi}{M}\right)$ are the shifted Chebyshev points (SCH-points).

From Eq. (7) and Eq. (8), we get

$$
\phi(x)=\sum_{r=0}^{M} \frac{2 c_{r}}{M} \sum_{m=0}^{M} c_{m} \mathcal{T}_{m}^{*}\left(x_{r}\right) \mathcal{T}_{m}^{*}(x) \phi\left(x_{r}\right) .
$$

According to El-Gendi [37], from Eq. (9),

$$
\int_{0}^{x_{s}} \phi(x) d x=\sum_{r=0}^{M} b_{s r} \phi\left(x_{r}\right), \quad s=0,1, \ldots, M,
$$

where

$$
b_{s r}=\frac{2 c_{r}}{M} \sum_{m=0}^{M} c_{m} \mathcal{T}_{m}^{*}\left(x_{r}\right) \int_{0}^{x_{s}} \mathcal{T}_{m}^{*}(x) d x, \quad s, r=0,1, \ldots, M
$$

are the elements of the B-matrix. Equation (10) and Eq. (11) can be written in the matrix form:

$$
\left[\int_{0}^{x} \phi(x) d x\right]=B \Phi
$$

such that

$$
B=\left[\begin{array}{cccc}
0 & 0 & \cdots & 0 \\
b_{10} & b_{11} & \cdots & b_{1 M} \\
\vdots & \vdots & \cdots & \vdots \\
b_{M 0} & b_{M 1} & \cdots & b_{M M}
\end{array}\right] \text { and } \Phi=\left[\begin{array}{c}
\phi\left(x_{0}\right) \\
\phi\left(x_{1}\right) \\
\vdots \\
\phi\left(x_{M}\right)
\end{array}\right]
$$

In the next section, some important theorems and lemmas are investigated. These theorems are needed in the process of SU-pseudo-GM. As mentioned before, this method is used to solve several types of fractional problems.

\section{Shifted ultraspherical pseudo-Galerkin method (SU-pseudo-GM)}

The first step, some rules and forms of UPs need to be shifted. Rodrigueś (Eq. (4)) formula is generalized in the shifted form

$$
\mathcal{U}_{j}^{* v}(x)=(-1)^{j} \frac{\Gamma\left(v+\frac{1}{2}\right) \Gamma(j+2 v)}{\Gamma(j+1) \Gamma(2 v) \Gamma\left(j+v+\frac{1}{2}\right)}\left(x-x^{2}\right)^{\frac{1}{2}-v} \frac{d^{j}}{d x^{j}}\left(x-x^{2}\right)^{j+v-\frac{1}{2}} .
$$

Thus, SUPs can be obtained from Eq. (5) or Eq. (12). The next lemma will be used to introduce a general form for the fractional derivative and the integer order integration of SUPs.

Lemma 1 The analytic form of SUPs is as follows:

$$
\mathcal{U}_{j}^{* v}(x)=\sum_{r=0}^{\lfloor j / 2\rfloor} \sum_{k=0}^{j-2 r} \frac{(-1)^{j-r-k} 2^{j-2 r+k} \Gamma(j-r+v)}{\Gamma(v) \Gamma(r+1) \Gamma(k+1) \Gamma(j-2 r-k+1)} x^{k}, \quad j=0,1,2, \ldots
$$


Proof Straightforward using Eq. (3) and the binomial theorem.

The following corollary proves the fractional derivatives of SUPs in the Caputo sense.

Corollary 2 The fractional derivatives of SUPs in the Caputo sense of order $\delta$ are as follows:

$$
D^{\delta}\left(\mathcal{U}_{j}^{* \nu}(x)\right)= \begin{cases}0, & j=0,1, \ldots,\lceil\delta\rceil-1, \\ \sum_{r=0}^{\lfloor(j-\delta) / 2\rfloor} \sum_{k=\lceil\delta\rceil}^{j-2 r} G_{r k}, & j=\lceil\delta\rceil,\lceil\delta\rceil+1, \ldots,\end{cases}
$$

where $G_{r k}=(-1)^{j-r-k} \frac{2^{j-2 r+k} \Gamma(j-r+v)}{\Gamma(v) \Gamma(r+1) \Gamma(k-\delta+1) \Gamma(j-2 r-k+1)} x^{k-\delta}$.

Proof Using Lemma 1 and the results from Eq. (2).

The next theorem will establish the general form of the SUPs approximation of a function $\phi(x)$. This form is used in SU-pseudo-GM.

Theorem 3 Consider $\phi(x)$ to be an " $s+1$ " differentiable function. Then $\phi(x)$ can be approximated as

$$
\phi(x)=\sum_{m=0}^{\infty} \theta_{m} \mathcal{A}_{m} \mathcal{U}_{m}^{* \nu}(x)
$$

where $\left\{\mathcal{A}_{m}\right\}$ are unknown constants to be determined later and

$$
\theta_{m}= \begin{cases}4 v, & m=0 \\ 2 v+1, & m=1 \\ 1, & 2 \leq m\end{cases}
$$

Proof Let $\phi(x)$ be approximated as follows:

$$
\phi(x)=\sum_{m=0}^{\infty} \mathcal{A}_{m} \mathcal{U}_{m}^{* v}(x)
$$

So, by the assumption in the theorem,

$$
\phi^{(s)}(x)=\sum_{m=0}^{\infty} \mathcal{A}_{m}^{(s)} \mathcal{U}_{m}^{* v}(x)
$$

and

$$
\begin{aligned}
\phi^{(s+1)}(x) & =\sum_{m=0}^{\infty} \mathcal{A}_{m}^{(s+1)} \mathcal{U}_{m}^{* \nu}(x) \\
& =\mathcal{A}_{0}^{(s+1)} \mathcal{U}_{0}^{* \nu}(x)+\mathcal{A}_{1}^{(s+1)} \mathcal{U}_{1}^{* \nu}(x)+\sum_{m=2}^{\infty} \mathcal{A}_{m}^{(s+1)} \mathcal{U}_{m}^{* \nu}(x)
\end{aligned}
$$


Substituting by $\mathcal{U}_{0}^{* v}(x)$ and $\mathcal{U}_{1}^{* v}(x)$ and using Eq. (6), we obtain

$$
\begin{aligned}
\phi^{(s+1)}(x)= & \mathcal{A}_{0}^{(s+1)}+\mathcal{A}_{1}^{(s+1)} 2 v(2 x-1)-\mathcal{A}_{0}^{(s+1)} 4 v \\
& -\mathcal{A}_{1}^{(s+1)} 2 v(2 v+1)(2 x-1) \\
& +\sum_{m=1}^{\infty}\left(\mathcal{A}_{m-1}^{(s+1)} \frac{2 v+m-1}{2(m+v-1) m}\right. \\
& \left.-\mathcal{A}_{m+1}^{(s+1)} \frac{m+1}{2(m+v+1)(v+m)}\right) D \mathcal{U}_{m}^{(* v)}(x) .
\end{aligned}
$$

By differentiating Eq. (15) w.r.t. $x$, we have

$$
\phi^{(s+1)}(x)=\sum_{m=1}^{\infty} \mathcal{A}_{m}^{(s)} D \mathcal{U}_{m}^{* v}(x)
$$

Both of the equations (16) and (17) represent the $s+1$ derivative of $\phi(x)$. Thus the following equation must be satisfied:

$$
\mathcal{A}_{m}^{(s)}=\mathcal{A}_{m-1}^{(s+1)} \frac{2 v+m-1}{2(m+v-1) m}-\mathcal{A}_{m+1}^{(s+1)} \frac{m+1}{2(m+v+1)(v+m)},
$$

and choose a set of constants $\left\{\theta_{m}\right\}$ as defended in (14).

\section{SU-pseudo-GM for solving OFPs}

This section is divided into three subsections. Each section concerns solving a different type of OFP. Also, an algorithm for each problem has been added "Algorithm 1, Algorithm 2, and Algorithm 3".

Before proceeding to the techniques of the solution, the " $M+1$ " collection points must be chosen. Throughout this paper three spaces of points are used. The set of equally spaced points " $S_{1}$ ":

$$
S_{1}=\left\{x_{m}=\frac{m}{M}, m=0,1, \ldots, M\right\},
$$

while the second is Gauss quadrate points " $S_{2}$ ", i.e., the zeros of SUPs:

$$
S_{2}=\left\{x, \mathcal{U}_{M+1}^{* v}(x)=0\right\}
$$

The last set is the $\mathrm{SCH}$-points " $S_{3}$ ":

$$
S_{3}=\left\{x_{m}=0.5\left(1-\cos \frac{m \pi}{M}\right), m=0,1, \ldots, M\right\} .
$$

" $S_{3}$ " is used specially for solving FIDEs.

\subsection{Solving OFDEs by SU-pseudo-GM}

Consider OFDEs as follows:

$$
F\left(\eta_{0}(x), \eta_{1}(x), \ldots, \eta_{k}(x), \phi(x), D^{\delta_{1}} \phi(x), \ldots, D^{\delta_{r}} \phi(x)\right)=0, \quad x \in[0,1]
$$




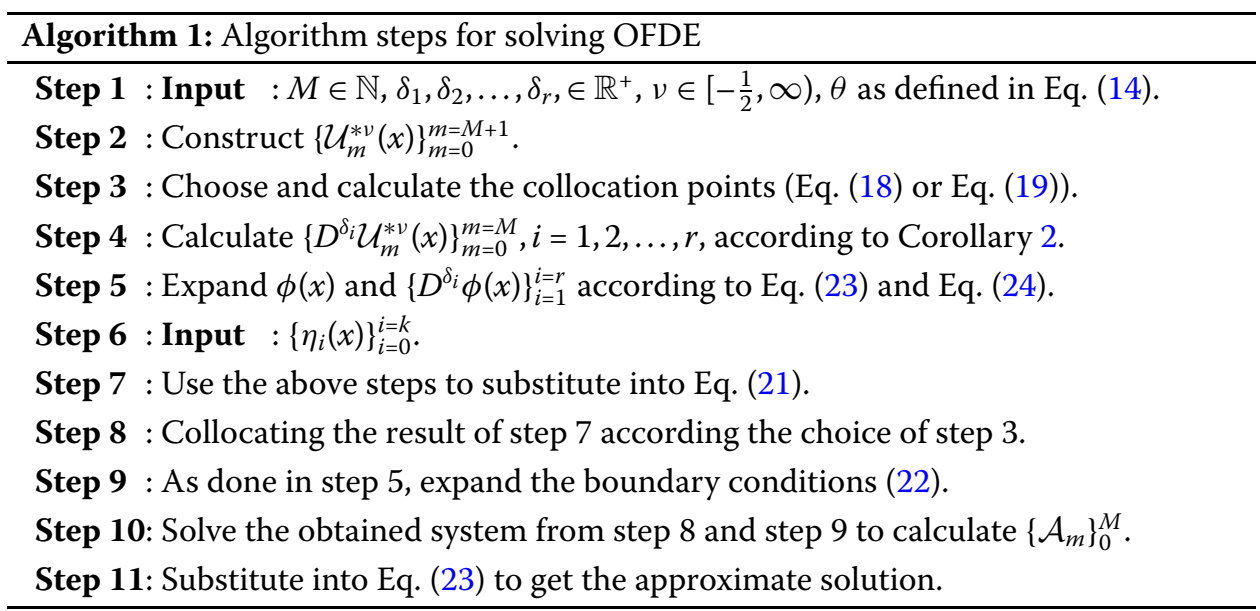

such that

$$
\left\{\begin{array}{l}
\phi^{j}(0)=d_{j}, \\
\phi^{q}(1)=h_{q}, \quad j, q=0, \ldots, r-1,
\end{array}\right.
$$

where $r, k \in \mathbb{Z}^{+},\left\{\delta_{i}\right\}_{1}^{r} \in \mathbb{R}^{+},\left\{\eta_{i}(x)\right\}_{0}^{k}$ are functions of $x$, the values of $d_{j}(i=0, \ldots, r-1)$ and $h_{q}(q=0, \ldots, r-1)$ describe the boundary or the initial state of $\phi(x)$. The linearity and the nonlinearity of OFDE (21) depend on the function $F$.

Now, by using SUPs as the base function in the spectral approximation of $\phi(x)$ as in Eq. (13), we have

$$
\phi(x)=\sum_{m=0}^{M} \theta_{m} \mathcal{A}_{m} \mathcal{U}_{m}^{* \nu}(x) .
$$

Using the straightforward differentiation in the sense of Corollary 2 leads to

$$
D^{\delta_{i}} \phi(x)=\phi^{\left(\delta_{i}\right)}(x)=\sum_{m=0}^{M} \theta_{m} \mathcal{A}_{m} D^{\delta_{i}} \mathcal{U}_{m}^{* \nu}(x)
$$

Also, the initial and boundary conditions (22) have series expansion of the form

$$
\left\{\begin{array}{l}
\sum_{m=0}^{M} \theta_{m} \mathcal{A}_{m} D^{i} \mathcal{U}_{m}^{* \nu}(0)=d_{j}, \\
\sum_{m=0}^{M} \theta_{m} \mathcal{A}_{m} D^{q} \mathcal{U}_{m}^{* \nu}(0)=h_{q},
\end{array} \quad j, q=0, \ldots, r-1\right.
$$

Substitute from Eqs. (23) and (24) into (21) by choosing $S_{1}$ or $S_{2}$ as nodes to get a system of linear/nonlinear algebraic equations together with Eq. (25). The unknowns of this system are $\left\{\mathcal{A}_{m}\right\}_{0}^{M}$. The system can be easily solved by any method. 


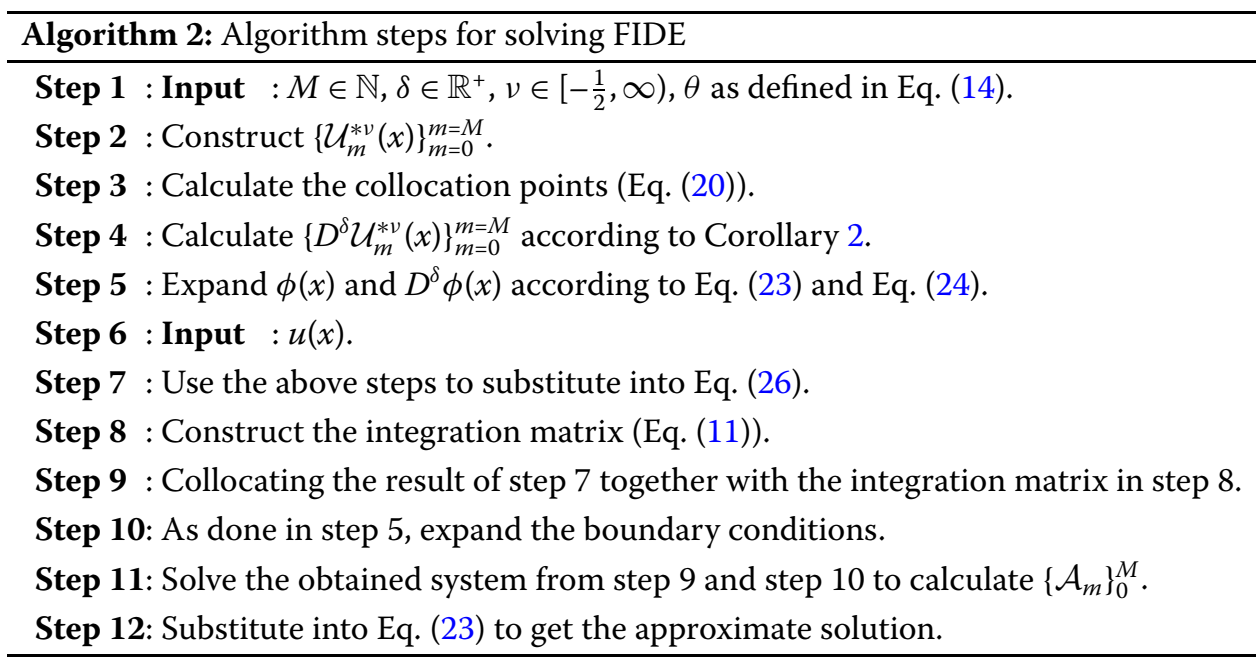

\subsection{Solving FIDEs by SU-pseudo-GM}

Herein, the SU-pseudo-GM for solving OFDEs is extended to some types of FIDEs. Consider the following FIDE:

$$
D^{\delta} \phi(x)=u(x)+\int_{0}^{x} k(x, t) F(\phi(t)) d t, \quad x \in[0,1],
$$

under a sufficient number of initial conditions according to $\delta$. By applying the expansion described at Eq. (23), we obtain

$$
\sum_{m=0}^{M} \theta_{m} \mathcal{A}_{m} D^{\delta} \mathcal{U}_{m}^{* \nu}\left(x_{s}\right)=u\left(x_{s}\right)+\int_{0}^{x_{s}} k\left(x_{s}, t\right) F\left(\sum_{m=0}^{M} \theta_{m} \mathcal{A}_{m} \mathcal{U}_{m}^{* \nu}(t)\right) d t
$$

where $s=0,1, \ldots, M$. Replacing the integration in Eq. (27) by the B-matrix (10) gives

$$
\sum_{m=0}^{M} \theta_{m} \mathcal{A}_{m} D^{\delta} \mathcal{U}_{m}^{* v}\left(x_{s}\right)=u\left(x_{s}\right)+\sum_{r=0}^{M} b_{s r} k\left(x_{s}, t_{r}\right) F\left(\sum_{m=0}^{M} \theta_{m} \mathcal{A}_{m} \mathcal{U}_{m}^{* \nu}\left(t_{r}\right)\right)
$$

As in the previous section, the obtained Eq. (28) is an algebraic system. The linearity of that system depends on the linearity of the function $F$.

\subsection{Solving FOCPs by SU-pseudo-GM}

Finally, we proceed to approximating the FOCP that takes the form

$$
\max (\min ) \quad \mathcal{J}(u)=\int_{0}^{1} F(x, \phi(x), u(x)) d x
$$

under the conditions

$$
\begin{aligned}
& D^{\delta} \phi(x)=G((x, \phi(x), u(x))) \\
& \phi(0)=c_{1}, \quad \phi^{\prime}(0)=c_{2},
\end{aligned}
$$




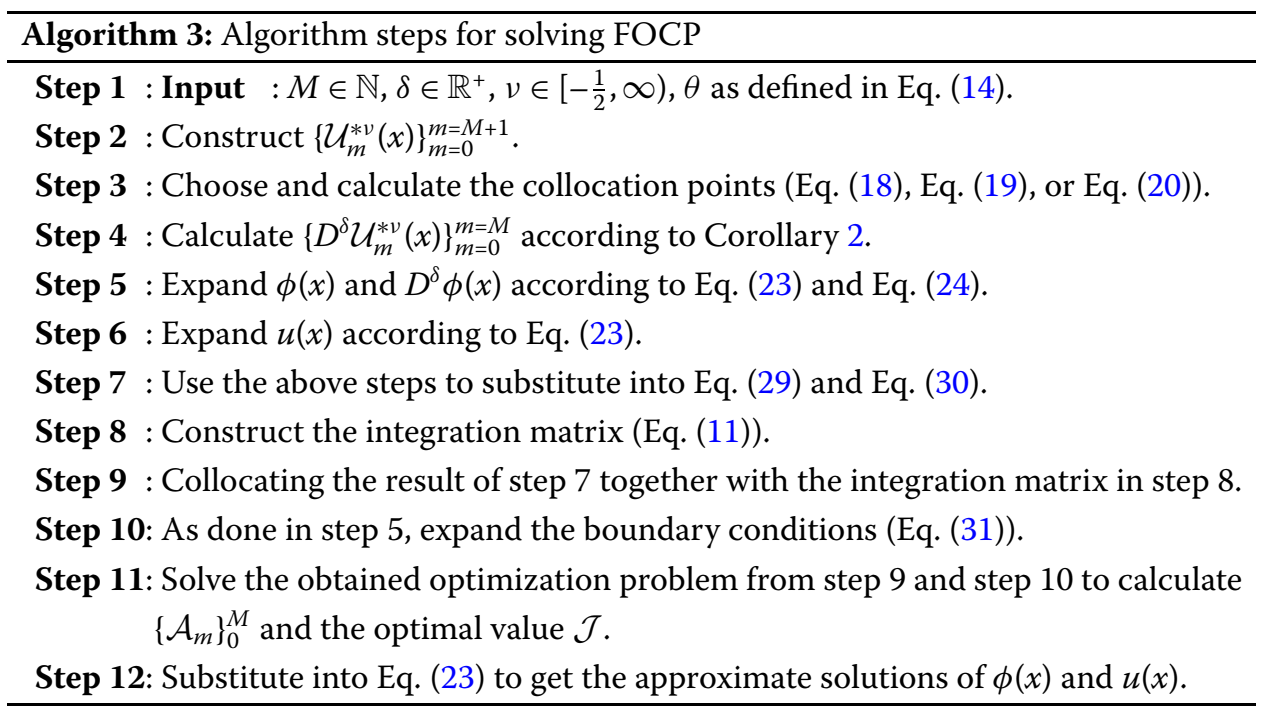

where $c_{1}, c_{2} \in \mathbb{R}$. By taking both techniques that are included in the last two subsections, the FOCP is transformed into a regular optimization problem.

\section{Error analysis}

The convergence and the error analysis for using SUPs are inevitable. Theorems and concepts for these aspects are investigated in $[34,35]$.

Lemma 4 Let $\phi(x) \in L_{w^{v}}^{2}[0,1],\left|\phi^{\prime \prime}(x)\right| \leq B$, and it can be expanded according to expansion (13). Then

$$
\left|\mathcal{A}_{m}\right|<\frac{4 B(1+v)^{2}(m+1+v)^{2}}{(m-2)^{4}}, \quad m \geq 3,
$$

where $0<v<1$.

Proof For the proof, see [38].

Theorem 5 Let $\phi_{M}(x)=\sum_{m=0}^{M} \theta_{m} \mathcal{A}_{m} \mathcal{U}_{m}^{* v}(x)$ and $\phi(x)$, v satisfies Lemma 4. Then

$$
\left\|\phi-\phi_{M}\right\|<\frac{B(1+v)^{2}(M+v)}{(M-3)^{7 / 2}}, \quad m \geq 3 .
$$

Proof See [38].

The next section proves the effectiveness and correctness of SU-pseudo-GM by solving different types of OFPs.

\section{Numerical examples}

In this section, numerical examples of SU-pseudo-GM are presented. The results to those obtained are compared with other methods and the exact solution. 
Table 1 The MAE for Example 1

\begin{tabular}{|c|c|c|c|c|c|c|c|}
\hline \multirow[t]{3}{*}{ c } & \multirow[t]{3}{*}{$M$} & \multicolumn{4}{|c|}{ SU-pseudo-GM } & \multirow[t]{3}{*}{ [39] } & \multirow[t]{3}{*}{ [40] } \\
\hline & & \multirow[t]{2}{*}{$\bar{S}$} & \multicolumn{3}{|l|}{$v$} & & \\
\hline & & & -0.49 & 0.49 & 1 & & \\
\hline \multirow[t]{3}{*}{1} & 8 & $\begin{array}{l}S_{1} \\
S_{2}\end{array}$ & $\begin{array}{l}6.2 \mathrm{e}-08 \\
2.5 \mathrm{e}-08\end{array}$ & $\begin{array}{l}6.2 \mathrm{e}-08 \\
4.3 \mathrm{e}-08\end{array}$ & $\begin{array}{l}6.2 \mathrm{e}-08 \\
3.1 \mathrm{e}-08\end{array}$ & $6.2 e-03$ & $2.0 e-12$ \\
\hline & 16 & $\begin{array}{l}S_{1} \\
S_{2}\end{array}$ & $\begin{array}{l}4.9 e-15 \\
2.7 e-15\end{array}$ & $\begin{array}{l}1.2 \mathrm{e}-14 \\
2.2 \mathrm{e}-15\end{array}$ & $\begin{array}{l}4.9 e-15 \\
1.3 e-15\end{array}$ & $7.5 \mathrm{e}-04$ & 4.0e-14 \\
\hline & 32 & $\begin{array}{l}S_{1} \\
S_{2}\end{array}$ & $\begin{array}{l}3.1 e-15 \\
2.7 e-15\end{array}$ & $\begin{array}{l}6.5 e-13 \\
2.2 e-15\end{array}$ & $\begin{array}{l}1.9 e-12 \\
1.3 e-15\end{array}$ & $1.0 \mathrm{e}-04$ & $2.0 e-11$ \\
\hline \multirow[t]{2}{*}{6} & 16 & $\begin{array}{l}S_{1} \\
S_{2}\end{array}$ & $\begin{array}{l}6.3 e-06 \\
2.1 e-07\end{array}$ & $\begin{array}{l}6.3 e-06 \\
3.6 e-07\end{array}$ & $\begin{array}{l}6.3 e-06 \\
2.0 e-07\end{array}$ & $8.4 \mathrm{e}-03$ & $1.0 \mathrm{e}-08$ \\
\hline & 32 & $\begin{array}{l}S_{1} \\
S_{2}\end{array}$ & $\begin{array}{l}6.9 \mathrm{e}-10 \\
9.4 \mathrm{e}-11\end{array}$ & $\begin{array}{l}1.4 \mathrm{e}-08 \\
5.1 \mathrm{e}-09\end{array}$ & $\begin{array}{l}1.3 e-09 \\
3.5 e-11\end{array}$ & $7.5 \mathrm{e}-04$ & $2.0 e-08$ \\
\hline
\end{tabular}

Table 2 The best MAE for Example 1

\begin{tabular}{llll}
\hline c & Method & Best MAE & M \\
\hline 1 & SU-pseudo-GM & $8.9 \mathrm{e}-16$ & 13 \\
& {$[39]$} & $1.6 \mathrm{e}-05$ & 64 \\
& {$[40]$} & $4.0 \mathrm{e}-14$ & 16 \\
6 & SU-pseudo-GM & $5.5 \mathrm{e}-12$ & 20 \\
& {$[39]$} & $6.5 \mathrm{e}-05$ & 64 \\
& {$[40]$} & $1.0 \mathrm{e}-08$ & 16 \\
\hline
\end{tabular}

Example 1 Consider the linear OFDE

$$
D^{2.5} \phi(x)-3 D^{2 / 3} \phi(x)=u(x),
$$

subject to $\phi(0)=1, \phi^{\prime}(0)=c$, and $\phi^{\prime \prime}(0)=c^{2}$ with the exact solution $\phi(x)=e^{c x}$ and $u(x)=$ $\frac{c^{2 / 3} e^{c x}\left[3 \Gamma(1 / 3, c x)+\left(c^{11 / 6} \operatorname{erf}(\sqrt{c x})-3\right) \Gamma(1 / 3)\right]}{\Gamma(1 / 3)}$.

According to Sect. 4.1, the maximum absolute error (MAE) for SU-pseudo-GM and MAE for others are presented in Table 1 and Table 2. These tables show the demonstration of SU-pseudo-GM over the methods in [39] and [40] for all values of $c$ and the parameter $v$ for the two spaces $S_{1}$ and $S_{2}$ at different values of $M$, while Fig. 1 represents the convergence at an exponential rate using $S_{3}$.

Example 2 Consider the nonlinear OFDE

$$
D^{\delta} \phi(x)+x(\phi(x))^{2}=x^{6}+\frac{\Gamma(3.5)}{\Gamma(3.5-\delta)} x^{2.5-\delta}
$$

subject to $\phi(0)=0$ with the exact solution $\phi(x)=x^{2.5}$.

Applying the technique discussed in Sect. 4.1, we obtained the pointwise absolute error (PW-AE) shown in Table 3 for $\delta=0.5$ and Table 4 for $\delta=0.7$. Comparisons between SU-pseudo-GM "at different values of $v$ ", the neural network method (NNM) in [41], the differentiated radial basis function method (DRBF) in [42], and the integrated radial basis function method (IRBF) in [42] have been done. From both tables we recognize that:

- SU-pseudo-GM is more accurate and efficient than NNM in [41] for the presented values of the parameter $\nu, \delta=0.5,0.7$ and both spaces $S_{1}$ and $S_{2}$. 


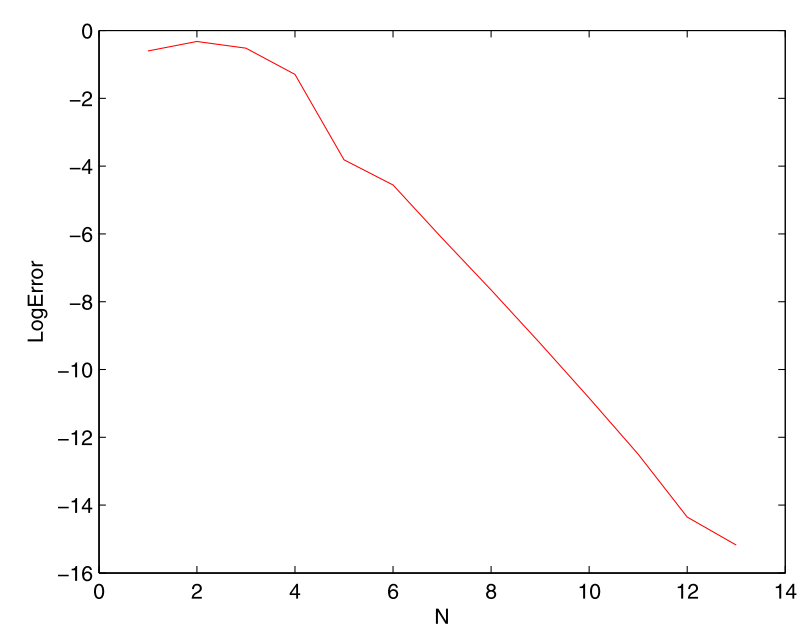

Figure 1 The LogError for Example 1 using $S_{3}$

Table 3 The PW-AE for Example 2 at $M=10$ and $\delta=0.5$

\begin{tabular}{|c|c|c|c|c|c|c|c|}
\hline \multirow[t]{3}{*}{$x$} & \multicolumn{4}{|c|}{ SU-pseudo-GM } & \multirow{3}{*}{$\begin{array}{l}\text { NNM } \\
{[41]}\end{array}$} & \multirow{3}{*}{$\begin{array}{l}\text { IRBF } \\
{[42]}\end{array}$} & \multirow{3}{*}{$\begin{array}{l}\text { DRBF } \\
{[42]}\end{array}$} \\
\hline & \multirow[t]{2}{*}{$\bar{S}$} & \multicolumn{3}{|l|}{$v$} & & & \\
\hline & & -0.49 & 0.49 & 1 & & & \\
\hline \multirow[t]{2}{*}{$\overline{0.1}$} & $S_{1}$ & $2.2 \mathrm{e}-05$ & $2.2 \mathrm{e}-05$ & $2.2 \mathrm{e}-05$ & $3.4 \mathrm{e}-03$ & $1.5 \mathrm{e}-05$ & $2.8 \mathrm{e}-05$ \\
\hline & $S_{2}$ & $7.8 \mathrm{e}-07$ & $1.6 \mathrm{e}-06$ & 7.7e-07 & & & \\
\hline \multirow[t]{2}{*}{0.2} & $S_{1}$ & $1.3 e-05$ & $1.3 e-05$ & $1.3 e-05$ & $8.7 e-03$ & $8.5 \mathrm{e}-06$ & $2.0 \mathrm{e}-05$ \\
\hline & $S_{2}$ & $2.5 \mathrm{e}-06$ & $1.3 e-06$ & $3.0 e-07$ & & & \\
\hline \multirow[t]{2}{*}{0.3} & $S_{1}$ & $1.0 \mathrm{e}-05$ & $1.0 \mathrm{e}-05$ & 1.0e-05 & $1.1 e-02$ & - & - \\
\hline & $S_{2}$ & $2.3 e-07$ & $1.5 \mathrm{e}-06$ & $1.1 \mathrm{e}-06$ & & & \\
\hline \multirow[t]{2}{*}{0.4} & $S_{1}$ & $8.7 e-06$ & 8.7e-06 & $8.7 e-06$ & $8.1 e-03$ & - & - \\
\hline & $\mathrm{S}_{2}$ & $1.2 \mathrm{e}-06$ & $2.8 \mathrm{e}-06$ & $2.1 \mathrm{e}-06$ & & & \\
\hline \multirow[t]{2}{*}{0.5} & $S_{1}$ & $7.5 e-06$ & $7.5 \mathrm{e}-06$ & $7.5 \mathrm{e}-06$ & $4.2 \mathrm{e}-04$ & 4.7e-06 & $6.7 \mathrm{e}-06$ \\
\hline & $S_{2}$ & $9.8 \mathrm{e}-07$ & $1.7 \mathrm{e}-06$ & $1.1 \mathrm{e}-06$ & & & \\
\hline \multirow[t]{2}{*}{0.6} & $S_{1}$ & $6.4 \mathrm{e}-06$ & $6.4 \mathrm{e}-06$ & $6.4 \mathrm{e}-06$ & $7.8 \mathrm{e}-03$ & $4.8 \mathrm{e}-06$ & $6.8 \mathrm{e}-06$ \\
\hline & $\mathrm{S}_{2}$ & $2.2 \mathrm{e}-08$ & $1.1 \mathrm{e}-06$ & $5.4 \mathrm{e}-07$ & & & \\
\hline \multirow[t]{2}{*}{0.7} & $S_{1}$ & $5.3 e-06$ & $5.3 e-06$ & $5.3 e-06$ & $9.1 \mathrm{e}-03$ & - & - \\
\hline & $S_{2}$ & $7.8 \mathrm{e}-07$ & $2.1 \mathrm{e}-06$ & $1.5 \mathrm{e}-06$ & & & \\
\hline \multirow[t]{2}{*}{0.8} & $S_{1}$ & $4.2 \mathrm{e}-06$ & 4.2e-06 & $4.2 e-06$ & $1.4 \mathrm{e}-03$ & - & - \\
\hline & $S_{2}$ & $3.6 \mathrm{e}-07$ & $1.7 \mathrm{e}-06$ & $1.3 e-06$ & & & \\
\hline \multirow[t]{2}{*}{0.9} & $S_{1}$ & $3.3 e-06$ & $3.3 e-06$ & $3.3 e-06$ & $1.6 \mathrm{e}-02$ & $1.5 \mathrm{e}-06$ & $1.2 \mathrm{e}-05$ \\
\hline & $\mathrm{S}_{2}$ & $2.3 e-07$ & $1.2 \mathrm{e}-06$ & $1.2 \mathrm{e}-06$ & & & \\
\hline \multirow[t]{2}{*}{1} & $S_{1}$ & $1.9 \mathrm{e}-06$ & $1.9 \mathrm{e}-06$ & $1.9 \mathrm{e}-06$ & $5.6 \mathrm{e}-03$ & $2.0 \mathrm{e}-06$ & 7.0e-06 \\
\hline & $S_{2}$ & $1.5 \mathrm{e}-07$ & $1.4 \mathrm{e}-06$ & $3.3 e-06$ & & & \\
\hline
\end{tabular}

- Using the equidistance space $S_{1}, \mathrm{SU}$-pseudo-GM is of almost the same accuracy as DRBF and IRBF methods in [42] for the presented values of the parameter $v$ and $\delta=0.5,0.7$.

- For $\delta=0.5$, SU-pseudo-GM got the same accuracy as DRBF and IRBF methods in [42] at $v=0.49$ using the $S_{2}$ space.

- For $\delta=0.5$, SU-pseudo-GM is more accurate than DRBF and IRBF methods in [42] for $v=1$ and $v=-0.49$ using the $S_{2}$ space.

- For $\delta=0.7$, SU-pseudo-GM is more accurate and efficient than DRBF and IRBF methods in [42] for the presented values of the parameter $v$ using the $S_{2}$ space.

- The values of the parameter $v$ do not affect the results while using $S_{1}$. 
Table 4 The pointwise AE for Example 2 at $M=10$ and $\delta=0.7$

\begin{tabular}{|c|c|c|c|c|c|c|c|}
\hline \multirow[t]{3}{*}{$x$} & \multicolumn{4}{|c|}{ SU-pseudo-GM } & \multirow{3}{*}{$\begin{array}{l}\text { NNM } \\
{[41]}\end{array}$} & \multirow{3}{*}{$\begin{array}{l}\text { IRBF } \\
{[42]}\end{array}$} & \multirow{3}{*}{$\begin{array}{l}\text { DRBF } \\
{[42]}\end{array}$} \\
\hline & \multirow[t]{2}{*}{$\bar{S}$} & \multicolumn{3}{|l|}{$v$} & & & \\
\hline & & $\overline{-0.49}$ & 0.49 & 1 & & & \\
\hline \multirow[t]{2}{*}{0.1} & $S_{1}$ & $4.5 \mathrm{e}-05$ & $4.5 e-05$ & $4.5 e-05$ & $2.3 e-03$ & $5.8 \mathrm{e}-05$ & $7.1 e-05$ \\
\hline & $S_{2}$ & $3.9 \mathrm{e}-06$ & $1.1 \mathrm{e}-06$ & $9.4 \mathrm{e}-07$ & & & \\
\hline \multirow[t]{2}{*}{0.2} & $S_{1}$ & $3.6 \mathrm{e}-05$ & 3.6e-05 & 3.6e-05 & $5.5 e-03$ & $4.4 \mathrm{e}-05$ & $5.5 e-05$ \\
\hline & $\mathrm{S}_{2}$ & $1.1 \mathrm{e}-07$ & $1.1 \mathrm{e}-06$ & $5.9 \mathrm{e}-07$ & & & \\
\hline \multirow[t]{2}{*}{0.3} & $S_{1}$ & $2.9 e-05$ & $2.9 e-05$ & $2.9 e-05$ & $7.3 e-03$ & - & - \\
\hline & $S_{2}$ & $2.0 e-07$ & $1.7 \mathrm{e}-06$ & $6.2 \mathrm{e}-07$ & & & \\
\hline \multirow[t]{2}{*}{0.4} & $S_{1}$ & $2.7 e-05$ & 2.7e-05 & $2.7 e-05$ & $6.3 e-03$ & - & - \\
\hline & $\mathrm{S}_{2}$ & $4.6 \mathrm{e}-06$ & $2.6 \mathrm{e}-06$ & $2.3 e-06$ & & & \\
\hline \multirow[t]{2}{*}{0.5} & $S_{1}$ & $2.4 \mathrm{e}-05$ & $2.4 \mathrm{e}-05$ & $2.4 \mathrm{e}-05$ & $1.5 e-03$ & $3.1 \mathrm{e}-05$ & $3.6 \mathrm{e}-05$ \\
\hline & $\mathrm{S}_{2}$ & $3.7 e-07$ & $1.6 \mathrm{e}-06$ & $3.8 \mathrm{e}-07$ & & & \\
\hline \multirow[t]{2}{*}{0.6} & $S_{1}$ & 2.2e-05 & 2.2e-05 & $2.2 \mathrm{e}-05$ & $5.6 \mathrm{e}-03$ & $2.8 \mathrm{e}-05$ & $3.9 e-05$ \\
\hline & $\mathrm{S}_{2}$ & $6.7 \mathrm{e}-07$ & $1.4 \mathrm{e}-06$ & $2.9 e-07$ & & & \\
\hline \multirow[t]{2}{*}{0.7} & $S_{1}$ & $1.9 \mathrm{e}-05$ & $1.9 \mathrm{e}-05$ & $1.9 \mathrm{e}-05$ & $9.0 \mathrm{e}-03$ & - & - \\
\hline & $S_{2}$ & $3.4 \mathrm{e}-06$ & $1.9 \mathrm{e}-06$ & $1.7 e-06$ & & & \\
\hline \multirow[t]{2}{*}{0.8} & $S_{1}$ & $1.6 \mathrm{e}-05$ & $1.6 e-05$ & $1.6 e-05$ & $1.2 \mathrm{e}-03$ & - & - \\
\hline & $S_{2}$ & $1.6 \mathrm{e}-07$ & $1.4 \mathrm{e}-06$ & $6.8 \mathrm{e}-07$ & & & \\
\hline \multirow[t]{2}{*}{0.9} & $S_{1}$ & $1.3 e-05$ & $1.3 e-05$ & $1.3 e-05$ & $1.5 \mathrm{e}-02$ & $1.5 \mathrm{e}-05$ & $2.3 e-05$ \\
\hline & $\mathrm{S}_{2}$ & $7.4 \mathrm{e}-07$ & 7.0e-07 & $1.1 \mathrm{e}-06$ & & & \\
\hline \multirow[t]{2}{*}{1} & $s_{1}$ & $9.7 e-06$ & $9.7 e-06$ & $9.7 e-06$ & $1.1 \mathrm{e}-02$ & $1.2 \mathrm{e}-05$ & $1.3 e-05$ \\
\hline & $\mathrm{S}_{2}$ & $1.1 \mathrm{e}-07$ & $3.8 \mathrm{e}-07$ & $1.7 \mathrm{e}-06$ & & & \\
\hline
\end{tabular}

During the process of approximation, we recognized that the results were not affected by changing the values of parameters $v$ at the small values of $M$.

Example 3 Consider the Bagley-Torvik fractional BVP

$$
D^{2} \phi(x)+D^{1.5} \phi(x)+\phi(x)=x^{3}+5 x+\frac{8}{\sqrt{\pi}} x^{1.5}, \quad x \in[0,1]
$$

subject to $\phi(0)=0$ and $\phi(1)=0$ with the exact solution $\phi(x)=-x+x^{3}$.

SU-pseudo-GM reaches the double-precision (e-16) as MAE only at $M=3$ using any space. While in [43], MAE varies from e- 04 to e- 05 by increasing $M$ from 10 to 40.

On the other hand, the authors in [44] investigated four methods. They treated the same problem with the first and fourth methods. The authors had to increase $M$ to 256 . But they only reached e-08 for the first method and e-05 for the fourth method.

Example 4 Consider the linear FIDE (Volterra type)

$$
D^{1 / 3} \phi(x)=-\frac{2}{9} x^{13 / 2}-\frac{2}{7} x^{9 / 2}+\frac{3 \sqrt{\pi}}{4 \Gamma(13 / 6)} x^{7 / 6}+\int_{0}^{x}\left(x y+(x y)^{2}\right) \phi(y) d y
$$

subject to $\phi(0)=0$ with the exact solution $\phi(x)=x^{1.5}$.

In this example, we have to use the space $S_{3}$ to meet the conditions of the B-matrix. The method in [45] solved Eq. (32) and got 9.5e-04 as MAE at $M=5$. Also, [46] solved it at the same number of points but by three methods. MAE were $1.9 \mathrm{e}-02,9.8 \mathrm{e}-0.3$, and 9.8e- 0.3 for the three methods. While the MAE of SU-pseudo-GM is 7.0e-04 using $M=5$ too. The authors in [46] increased M to increase the accuracy. Their best MAE was $10^{-4}$ at 
Table 5 The MAE for Example 4 using $S_{3}$

\begin{tabular}{|c|c|c|c|c|}
\hline \multirow[t]{2}{*}{ M } & \multirow[t]{2}{*}{ SU-pseudo-GM } & \multicolumn{2}{|l|}{$[46]$} & \multirow[t]{2}{*}[45]{} \\
\hline & & Method 1 & Method 2\&3 & \\
\hline 5 & 7.0e-0.4 & $2.0 e-02$ & $9.8 \mathrm{e}-03$ & $9.5 e-04$ \\
\hline 10 & $8.7 e-05$ & $6.3 e-03$ & $3.4 \mathrm{e}-03$ & - \\
\hline 20 & $1.1 \mathrm{e}-05$ & $2.1 \mathrm{e}-03$ & $1.2 \mathrm{e}-03$ & - \\
\hline
\end{tabular}

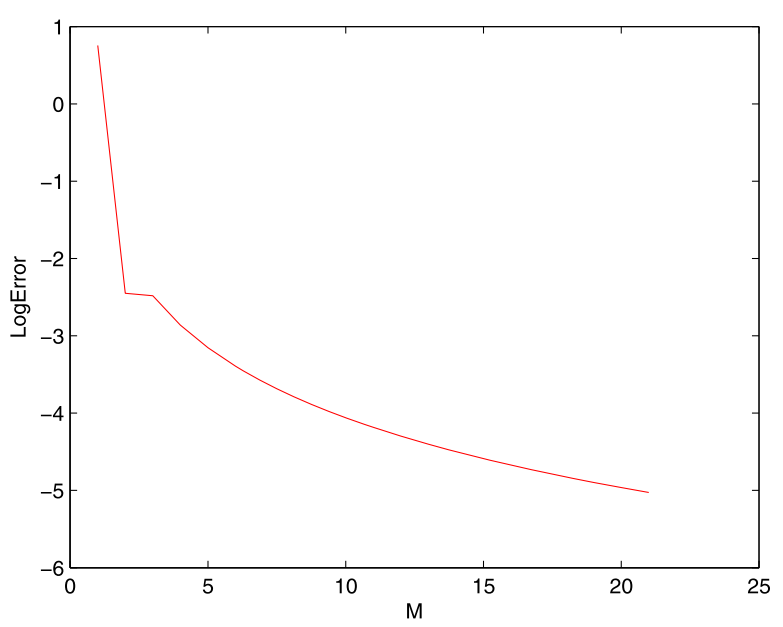

Figure 2 The LogError for Example 4 using $S_{3}$

$M=40,80$. But by using SU-pseudo-GM, MAE is 8.7e-05 at $M=10$ and $9.4 \mathrm{e}-06$ at $M=21$. Some results and comparisons are summarized in Table 5 . The stability and convergence rate are shown in Fig. 2.

Example 5 Consider the nonlinear FIDE (Volterra-Fredholm type)

$$
\begin{aligned}
D^{\sqrt{3}} \phi(x)= & -\frac{15}{56} x^{8}-\frac{1}{6} x^{2}+\frac{2(2+\sqrt{3})}{\Gamma(2-\sqrt{3})} x^{2-\sqrt{3}} \\
& +\int_{0}^{x}(x+y)(\phi(y))^{3} d y+\int_{0}^{1} x^{2} y(\phi(y))^{2} d y
\end{aligned}
$$

subject to the boundary conditions of mixed type $\phi(0)+\phi^{\prime}(0)=0$ and $\phi(1)+\phi^{\prime}(1)=3$ with the exact solution $\phi(x)=x^{2}$.

The boundary conditions of mixed type do not affect the steps. They are just two extra equations added to the algebraic system. The PW-AE of this example using SU-pseudoGM and two other methods in [47] is written in Table 6. The first method in [47] is "Nystrom", while the second method depends on the wavelet. This method used the two parameters $m$ and $k$ for the approximation. So, $2^{k-1} m$ is the equivalent number of iterations. The following observations have been noted from Table 6. This first method in [47] approximates the solution to $10^{-5}$ as PW-AE at $M=16$. But SU-pseudo-GM got the same $\mathrm{PW}-\mathrm{AE}$ at $M=5$ only. On the other hand, PW-AE decreases from $10^{-6}$ to $10^{-2}$ as $x$ changes

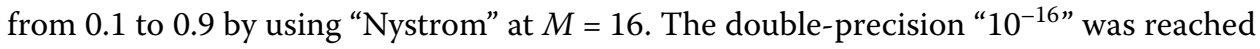


Table 6 The MAE for Example 5 using $S_{3}$

\begin{tabular}{|c|c|c|c|c|c|}
\hline \multirow[t]{3}{*}{$x$} & \multicolumn{3}{|c|}{ SU-pseudo-GM } & \multicolumn{2}{|l|}{$[47]$} \\
\hline & \multirow[t]{2}{*}{$M=5$} & \multirow[t]{2}{*}{$M=7$} & \multirow[t]{2}{*}{$M=20$} & Method 1 & Nystrom \\
\hline & & & & $M=16$ & $M=20$ \\
\hline 0 & $7.46 e-05$ & $1.76 e-15$ & $1.28 \mathrm{e}-15$ & $7.66 e-05$ & 0 \\
\hline 0.1 & $6.70 e-05$ & $1.59 e-15$ & $1.12 \mathrm{e}-15$ & $6.90 \mathrm{e}-05$ & $4.87 e-06$ \\
\hline 0.2 & $6.02 \mathrm{e}-05$ & $1.45 e-15$ & $1.05 e-15$ & $6.13 e-05$ & $6.50 \mathrm{e}-06$ \\
\hline 0.3 & $5.68 \mathrm{e}-05$ & $1.30 e-15$ & $9.44 e-16$ & $5.36 \mathrm{e}-05$ & $3.04 \mathrm{e}-05$ \\
\hline 0.4 & $5.70 e-05$ & $1.17 e-15$ & $8.33 e-16$ & $4.60 \mathrm{e}-05$ & $1.01 \mathrm{e}-04$ \\
\hline 0.5 & $5.91 e-05$ & $1.05 e-15$ & $7.77 e-16$ & $3.72 \mathrm{e}-05$ & $3.29 \mathrm{e}-04$ \\
\hline 0.6 & $6.09 e-05$ & $9.44 \mathrm{e}-16$ & $6.66 e-16$ & $3.07 e-05$ & $1.16 \mathrm{e}-03$ \\
\hline 0.7 & $6.02 \mathrm{e}-05$ & $8.33 e-16$ & $6.11 e-16$ & $2.22 \mathrm{e}-05$ & $4.13 e-03$ \\
\hline 0.8 & 5.59e-05 & $7.77 e-16$ & $4.44 \mathrm{e}-16$ & $1.36 \mathrm{e}-05$ & $1.39 \mathrm{e}-02$ \\
\hline 0.9 & $4.89 e-05$ & $7.77 e-16$ & $5.55 e-16$ & $6.90 \mathrm{e}-05$ & $4.40 e-02$ \\
\hline 1 & $4.26 e-05$ & $6.66 e-16$ & $4.44 \mathrm{e}-16$ & - & - \\
\hline
\end{tabular}

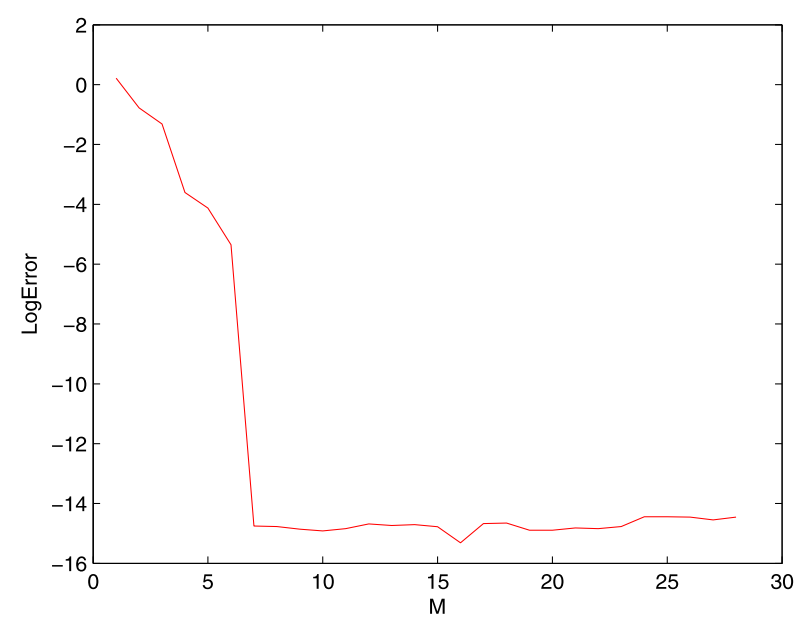

Figure 3 The LogError for Example 5 using $S_{3}$

using SU-pseudo-GM at $M=16$ for all the domain points. Figure 3 ensures the accuracy, stability, and convergence rate for SU-pseudo-GM.

Example 6 Consider the nonlinear FOCP

$$
(\min ) \quad \mathcal{J}=\int_{0}^{1}\left(\left(\phi(x)-x^{2}\right)^{2}-\left(v(x)+x^{4}-\frac{20 x^{0.9}}{9 \Gamma(0.9)}\right)^{2}\right) d x
$$

subject to $D^{1.1} \phi(x)=x^{2} \phi(x)+v(x)$ and $\phi(0)=\phi^{\prime}(0)=0$ with the exact solution $(\mathcal{J}(v), \phi(x)$, $v(x))=\left(0, x^{2}, \frac{20 x^{0.9}}{9 \Gamma(0.9)}-x^{4}\right)$.

According to the integration "from 0 to 1 ", all spaces" $S_{1}, S_{2}, S_{3}$ " may be used. The same technique is applied. In the past examples we got algebraic systems to solve. But in the case of FOCP, the problem is transformed into an optimization problem.

The best result is $\mathcal{J}=4.1280 e-17$ at " $M=2$ " when " $v=-0.49$ " using " $S_{2}$ " with run time 0.019 . The results of SU-pseudo-GM have been compared with the results from [18, 19,48 ]. In [18], the authors used wavelet expansion. They got $\mathcal{J}=4.7187 e-06$ using six 
Table 7 Optimality value " $\mathcal{J}$ ", MAE of the state function " $\phi(x)$ " and the control function " $v(x)$ " for Example 6 using $S_{1}$ when $v=0.49$

\begin{tabular}{|c|c|c|c|c|c|}
\hline \multirow[t]{3}{*}{$M$} & \multicolumn{4}{|c|}{ SU-pseudo-GM } & \multirow{3}{*}{$\frac{[48]}{\mathcal{J}(v)}$} \\
\hline & \multicolumn{2}{|l|}{$\overline{\mathcal{J}(v)}$} & \multirow[t]{2}{*}{$\phi(x)$} & \multirow[t]{2}{*}{$v(x)$} & \\
\hline & Value & Time & & & \\
\hline 2 & $4.9816 \mathrm{e}-16$ & 0.012 & $1.7155 \mathrm{e}-08$ & $2.7391 \mathrm{e}-08$ & - \\
\hline 3 & $7.5675 e-16$ & 0.021 & $2.5321 \mathrm{e}-08$ & $3.8533 e-08$ & - \\
\hline 4 & $4.3574 \mathrm{e}-16$ & 0.035 & $2.0878 \mathrm{e}-08$ & $4.8836 \mathrm{e}-08$ & $4.7693 e-06$ \\
\hline 5 & $4.5959 \mathrm{e}-16$ & 0.044 & $2.2785 e-08$ & $5.7676 \mathrm{e}-08$ & $1.4724 \mathrm{e}-06$ \\
\hline 6 & $8.4043 e-15$ & 0.071 & $2.4193 e-08$ & $1.5770 \mathrm{e}-07$ & $5.3783 e-07$ \\
\hline 7 & $6.7510 \mathrm{e}-13$ & 0.099 & $1.2375 \mathrm{e}-07$ & $5.6828 \mathrm{e}-06$ & - \\
\hline 8 & $8.7378 \mathrm{e}-13$ & 0.139 & $2.2888 \mathrm{e}-07$ & $3.5761 \mathrm{e}-06$ & $1.0610 \mathrm{e}-07$ \\
\hline 9 & $5.4106 \mathrm{e}-14$ & 0.181 & $5.4007 e-08$ & $5.7890 \mathrm{e}-07$ & $5.4430 \mathrm{e}-07$ \\
\hline
\end{tabular}

Table 8 Best results of Example 7

\begin{tabular}{|c|c|c|c|}
\hline \multirow{2}{*}{$\begin{array}{l}\text { Optimal value } \\
\mathcal{J}\end{array}$} & \multicolumn{2}{|l|}{ MAE } & \multirow[t]{2}{*}{ Method } \\
\hline & $\begin{array}{l}\text { State function } \\
\phi(x)\end{array}$ & $\begin{array}{l}\text { Control function } \\
v(x)\end{array}$ & \\
\hline $\begin{array}{l}10^{-17} \\
(M=3)\end{array}$ & $\begin{array}{l}10^{-16} \\
(M=3)\end{array}$ & $\begin{array}{l}10^{-09} \\
(M=3)\end{array}$ & $\overline{\text { SU-pseudo-GM }}$ \\
\hline $\begin{array}{l}10^{-16} \\
(M=4)\end{array}$ & - & - & [19] \\
\hline $\begin{array}{l}10^{-10} \\
(M=9)\end{array}$ & $\begin{array}{l}10^{-05} \\
(M=9) \text { "PW-AE" }\end{array}$ & $\begin{array}{l}10^{-01} \\
(M=9) \text { "PW-AE" }\end{array}$ & [18] \\
\hline $\begin{array}{l}10^{-05} \\
(M=7)\end{array}$ & $\begin{array}{l}10^{-03} \\
(M=7)\end{array}$ & $\begin{array}{l}10^{-05} \\
(M=7)\end{array}$ & [49] \\
\hline $\begin{array}{l}10^{-06} \\
(M=8)\end{array}$ & $\begin{array}{l}10^{-05} \\
(M=6) \text { "PW-AE" }\end{array}$ & - & {$[50]$} \\
\hline
\end{tabular}

iterations with run time 0.046 . While in [19], $\mathcal{J}=6.16 e-16$ but at $M=4$ with run time 0.030 and in [48], $\mathcal{J}=5.44 e-08$ at $M=9$ as best results. Table 7 compares the results obtained by SU-pseudo-GM when $v=0.49$ using $S_{1}$ with results from [48].

These comparisons show the accuracy and the efficiency of SU-pseudo-GM for solving FCOPs.

Example 7 Consider the nonlinear FOCP

$$
\begin{aligned}
& (\min ) \quad \mathcal{J}=\int_{0}^{1}\left(-2 e^{1+x^{2}+\phi(x)}+e^{2\left(1+x^{2}+\phi(x)\right)}+8 \sqrt{\frac{x}{\pi}} v(x)+(v(x))^{2}+\frac{16}{\pi} x\right. \\
& \left.-2 \sin \left(1+x^{2}\right) v(x)-8 \sqrt{\frac{x}{\pi}} \sin \left(1+x^{2}\right)+\sin ^{2}\left(1+x^{2}\right)+1\right) d x
\end{aligned}
$$

subject to $D^{1.5} \phi(x)=\sin (\phi(x))+v(x), \phi(0)=-1$, and $\phi^{\prime}(0)=0$ with the exact solution $(\mathcal{J}(v), \phi(x), v(x))=\left(0,-1-x^{2},-4 \sqrt{\frac{x}{\pi}}+\sin \left(1+x^{2}\right)\right)$.

By applying the same steps, Table 8 summarizes the best results of $\mathcal{J}$, MAE of the state function $\phi(x)$, and MAE of the control function $v(x)$. All those results are compared with the methods in $[18,19,49,50]$. The obtained results prove the high correctness and effectiveness of SU-pseudo-GM. Figure 4 shows the stability of the optimality value. 


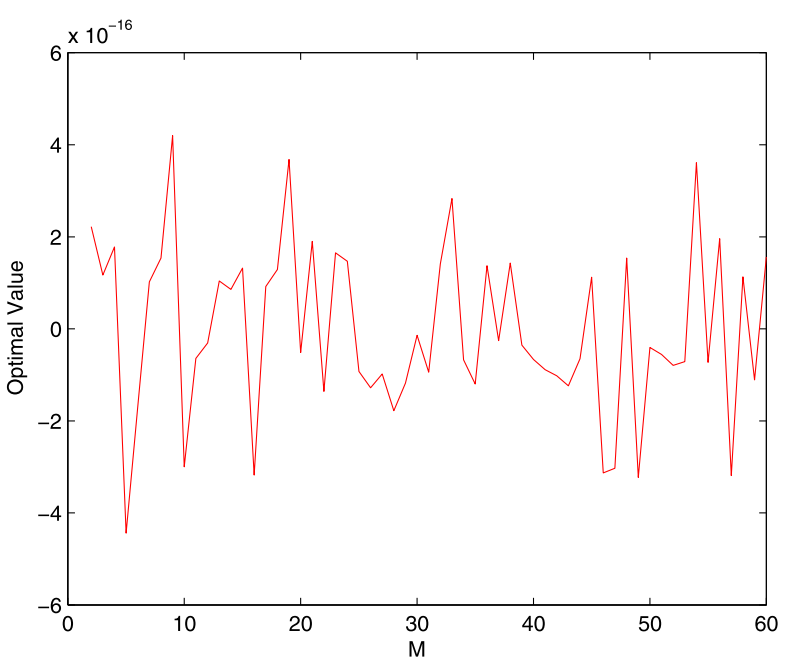

Figure 4 The optimal value $\mathcal{J}(x)$ for Example 7 using $S_{3}$ at $v=-0.49$

\section{Conclusion}

A general method developed from GM has been introduced in this work. This method uses SUPs as trails functions. We call this method SU-pseudo-GM. New formulae for the spectral expansion and fractional derivatives of SUPs have been proved. This method can handle several types of OFPs. Finally, SU-pseudo-GM was used to solve several examples. As shown in those examples, the method was easy to apply in FODEs, IFDEs, and FOCPs. To show and prove the high correctness of that method, several graphs have been constructed. Also, comparisons with other methods have been done. Three spaces have been used in the examples. The results show that the quadrature points $\left(S_{2}\right)$ are slightly more accurate than the equidistant Riemann points $\left(S_{1}\right)$.

\section{Acknowledgements}

The authors are sincerely grateful to Dr. Youssri H. Youssri, Associate Professor at Cairo University in Egypt, for his valuable support during this research.

Funding

The authors received no financial support for the research.

Availability of data and materials

All data are included within this paper.

Competing interests

The authors declare that they have no competing interests.

\section{Authors' contributions}

The first, third, and fourth authors participated equally in developing the idea and derivations. The second author participated in the derivation part. All authors read and approved the final manuscript.

\section{Author details}

'Department of Mathematics, Faculty of Science, Helwan University, Cairo, Egypt. ${ }^{2}$ Basic Science Department, School of Engineering, Canadian International College, New Cairo, Egypt. ${ }^{3}$ Department of Mathematics, Cankaya University, Ankara, Turkey. ${ }^{4}$ Institute of Space Sciences, Magurele-Bucharest, Romania. ${ }^{5}$ Department of Medical Research, China Medical University Hospital, China Medical University, Taichung, Taiwan.

\section{Publisher's Note}

Springer Nature remains neutral with regard to jurisdictional claims in published maps and institutional affiliations. 


\section{References}

1. Constantinescu, C.D., Ramirez, J.M., Zhu, W.R.: An application of fractional differential equations to risk theory. Finance Stoch. 23, 1001-1024 (2019)

2. Butzer, P.L., Westphal, U.: An introduction to fractional calculus. In: Hilfer, R. (ed.) Applications of Fractional Calculus in Physics, pp. 1-86. World Scientific, Singapore (2000)

3. Saad, K.M., Gómez-Aguilar, J.F., Almadiy, A.A.: A fractional numerical study on a chronic hepatitis $C$ virus infection model with immune response. Chaos Solitons Fractals 139, Article ID 110062 (2020)

4. Jajarmi, A., Arshad, S., Baleanu, D.: A new fractional modelling and control strategy for the outbreak of Dengue fever. Phys. A, Stat. Mech. Appl. 535, Article ID 122524 (2019)

5. Ionescu, C., Lopes, A., Copot, D., Machado, J.A.T., Bates, J.H.T.: The role of fractional calculus in modeling biological phenomena: a review. Commun. Nonlinear Sci. Numer. Simul. 51, 141-159 (2017)

6. Arafa, A.A.M., Rida, S.Z., Khalil, M.: Solutions of fractional order model of childhood diseases with constant vaccination strategy. Math. Sci. Lett. 1(1), 17-23 (2012)

7. Tarasov, V.E.: Fractional integro-differential equations for electromagnetic waves in dielectric media. Theor. Math. Phys. 158, 355-359 (2009)

8. Santamaría, G., Valverde, J., Pérez-Aloe, R., Vinagre, B.: Microelectronic implementations of fractional-order integro-differential operators. J. Comput. Nonlinear Dyn. 3(2), Article ID 021301 (2009)

9. Mohammad, M., Trounev, A.: Implicit Riesz wavelets based-method for solving singular fractional integro-differential equations with applications to hematopoietic stem cell modeling. Chaos Solitons Fractals 138, Article ID 109991 (2020)

10. AbdElal, L.F., Sweilam, N.H., Nagy, A.M., Almaghrebi, Y.S.: Computational methods for the fractional optimal control HIV infection. J. Fract. Calc. Appl. 7(2), 121-131 (2016)

11. Ameen, I., Baleanu, D., Ali, H.M.: An efficient algorithm for solving the fractional optimal control of SIRV epidemic model with a combination of vaccination and treatment. Chaos Solitons Fractals 137(2). Article ID 109892 (2020)

12. Abd-Elhameed, W.M., Youssri, Y.H.: Explicit shifted second-kind Chebyshev spectral treatment for fractional Riccati differential equation. Comput. Model. Eng. Sci. 121(3), 1029-1049 (2019)

13. Abd-Elhameed, W.M., Youssri, Y.H.: Sixth-kind Chebyshev spectral approach for solving fractional differential equations. Int. J. Nonlinear Sci. Numer. Simul. 20(2), 191-203 (2019)

14. Hamasalh, F.K., Muhammed, P.O.: Computational method for fractional differential equations using nonpolynomial fractional spline. Math. Lett. 5(2), 131-136 (2016)

15. Hamasalh, F.K., Muhammed, P.O.: Computational non-polynomial spline function for solving fractional Bagely-Torvik equation. Math. Lett. 6(1), 83-87 (2017)

16. Hanna, LM Al-Kandari, M. Luchko, Y. Operational method for solving fractional differential equations with the left-and right-hand sided Erdélyi-Kober fractional derivatives. Fract. Calc. Appl. Anal. 23(1), 103-125 (2020)

17. Rahimkhani, P., Ordokhani, Y.: Approximate solution of nonlinear fractional integro-differential equations using fractional alternative Legendre functions. J. Comput. Appl. Math. 365, Article ID 112365 (2020)

18. Rabiei, K., Ordokhani, Y.: A new operational matrix based on Boubaker wavelet for solving optimal control problems of arbitrary order. J. Vib. Control 42(10), 1858-1870 (2020)

19. Abdelhakem, M., Moussa, H., Baleanu, D., El-Kady, M.: Shifted Chebyshev schemes for solving fractional optimal control problems. J. Vib. Control 25(15), 2143-2150 (2019)

20. Abdelhakem, M., Ahmed, A., El-kady, M.: Spectral monic Chebyshev approximation for higher order differential equations. Math. Sci. Lett. 8(2), 11-17 (2019)

21. Abdelhakem, M., Biomy, B., Kandil, S.A., Baleanu, D., El-kady, M.: A numerical method based on Legendre differentiation matrices for higher order ODEs. Inf. Sci. Lett. 9(2), 175-180 (2020)

22. Doha, E.H., Abd-Elhameed, W.M., Youssri, Y.H.: New ultraspherical wavelets collocation method for solving 2nth-order initial and boundary value problems. J. Egypt. Math. Soc. 24(2), 319-327 (2016)

23. Youssri, Y.H., Abd-Elhameed, W.M., Doha, E.H.: Accurate spectral solutions of first- and second-order initial value problems by the ultraspherical wavelets-Gauss collocation method. Appl. Appl. Math. 10(2), $835-851$ (2015)

24. Abd-Elhameed, W.M., Youssri, Y.H., Doha, E.H.: New solutions for singular Lane-Emden equations arising in astrophysics based on shifted ultraspherical operational matrices of derivatives. Comput. Methods Differ. Equ. 2(3), 171-185 (2014)

25. Szegö, G.: Orthogonal Polynomials, 1st edn. Am. Math. Soc., Providence (1939)

26. Zaky, M.A.: An accurate spectral collocation method for nonlinear systems of fractional differential equations and related integral equations with nonsmooth solutions. Appl. Numer. Math. 154, 205-222 (2020)

27. Zaky, M.A., Hendy, A.S.: Convergence analysis of a Legendre spectral collocation method for nonlinear Fredholm integral equations in multidimensions. Math. Methods Appl. Sci. 1(14), Doi (2020). https://doi.org/10.1002/mma.6443

28. Zaky, M.A., Ameen, I.G.: On the rate of convergence of spectral collocation methods for nonlinear multi-order fractional initial value problems. Comput. Appl. Math. 38, 144 (2019)

29. Zaky, M.A., Hendy, A.S.: Convergence analysis of an $L_{1}$-continuous Galerkin method for nonlinear time-space fractional Schrödinger equations. Int. J. Comput. Math. (2020). https://doi.org/10.1080/00207160.2020.1822994

30. Zaky, M.A., Hendy, A.S., Macías-Díaz, J.E.: Semi-implicit Galerkin-Legendre spectral schemes for nonlinear time-space fractional diffusion-reaction equations with smooth and nonsmooth solutions. J. Sci. Comput. 28, 13 (2020)

31. Hendy, A.S., Zaky, M.A.: Global consistency analysis of L1-Galerkin spectral schemes for coupled nonlinear space-time fractional Schrödinger equations. Appl. Numer. Math. 156, 276-302 (2020)

32. Teodoro, G.S., Machado, J.A.T., de Oliveira, E.C.: A review of definitions of fractional derivatives and other operators. J. Comput. Phys. 388, 195-208 (2019)

33. Abramowitz, M., Stegun, I.A.: Handbook of Mathematical Functions. Dover, New York (1965)

34. Hafez, R.M., Youssri, Y.H.: Shifted Gegenbauer-Gauss collocation method for solving fractional neutral functional-differential equations with proportional delays. Kragujev. J. Math. 46(6), 981-996 (2022)

35. Ahmed, H.F., Melad, M.B.: New numerical approach for solving fractional differential- algebraic equations. J. Fract. Calc. Appl. 9(2), 141-162 (2018)

36. Shen, J., Tang, T., Wang, L.L.: Spectral Methods: Algorithms, Analysis and Applications. Springer, Berlin (2011) 
37. El-Gendi, S.E.: Chebyshev solution of differential, integral and integro-differential equations. Comput. J. 12(3), 282-287 (1969)

38. Youssri, Y.H., Abd-Elhameed, W.M., Doha, E.H.: Ultraspherical wavelets method for solving Lane-Emden type equations. Rom. J. Phys. 60(9-10), 1298-1341 (2015)

39. Doha, E.H., Bhrawy, A.H., Ezz-Eldien, S.S.: Efficient Chebyshev spectral methods for solving multi-term fractional orders differential equations. Appl. Math. Model. 35, 5662-5672 (2011)

40. Atta, A.G., Moatimid, G.M., Youssri, Y.H.: Generalized Fibonacci operational collocation approach for fractional initial value problems. Int. J. Appl. Comput. Math. 5, Article ID 9 (2019)

41. Qu, H., Liu, X.: A numerical method for solving fractional differential equations by using neural network. Adv. Math. Phys. 2015, Article ID 439526 (2015)

42. Chandhini, G., Prashanthi, K.S., Vijesh, V.A.: Direct and integrated radial functions based quasilinearization schemes for nonlinear fractional differential equations. BIT Numer. Math. 60, 31-65 (2020)

43. Sakar, M.G., Saldır, O., Akgül, A.: A novel technique for fractional Bagley-Torvik equation. Proc. Natl. Acad. Sci. India Sect. A Phys. Sci. 98(3), 539-545 (2019)

44. Emadifar, H., Jalilian, R.: An exponential spline approximation for fractional Bagley-Torvik equation. Bound. Value Probl. 2020, Article ID 20 (2020)

45. Varol Bayram, D., Daşcıoğlu, A.: A method for fractional Volterra integro-differential equations by Laguerre polynomials. Adv. Differ. Equ. 2018, Article ID 466 (2018)

46. Kumar, K., Pandey, R.K., Sharma, S.: Comparative study of three numerical schemes for fractional integro-differential equations. J. Comput. Appl. Math. 315, 287-302 (2017)

47. Ali, M.R., Hadhoud, A.R., Srivastava, H.M.: Solution of fractional Volterra-Fredholm integro-differential equations under mixed boundary conditions by using the HOBW method. Adv. Differ. Equ. 2019, Article ID 115 (2019)

48. Bhrawy, A.H., Doha, E.H., Baleanu, D., Ezz-Eldien, S.S., Abdelkawy, M.A.: An accurate numerical technique for solving fractional optimal control problems. Proc. Rom. Acad., Ser. A: Math. Phys. Tech. Sci. Inf. Sci. 16(1), 47-54 (2015)

49. Behroozifar, M., Habibi, N.: A numerical approach for solving a class of fractional optimal control problems via operational matrix Bernoulli polynomials. J. Vib. Control 24(12), 2494-2511 (2018)

50. Nemati, A., Yousefi, A.: A numerical method for solving fractional optimal control problems using Ritz method. J. Comput. Nonlinear Dyn. 11(5), Article ID 051015 (2016)

\section{Submit your manuscript to a SpringerOpen ${ }^{0}$ journal and benefit from:}

- Convenient online submission

- Rigorous peer review

- Open access: articles freely available online

- High visibility within the field

- Retaining the copyright to your article

Submit your next manuscript at $\gg$ springeropen.com 